\title{
Facial nerve injury immune-related adverse events associated with adjuvant pembrolizumab therapy: A case report
}

\author{
YAZHENG DANG $^{1 *}$, MINGWEI WANG $^{2 *}$, WEI LI $^{2 *}$, HONGXIANG GAO $^{3}$, SHIGAO HUANG $^{4}$ and TAO QI ${ }^{1}$ \\ ${ }^{1}$ Department of Radiation Oncology, 986 Hospital Affiliated to The Fourth Military Medical University, Xi'an, \\ Shaanxi 710054; ${ }^{2}$ Department of Radiotherapy, The Third People's Hospital Affiliated to Nantong University, \\ Nantong, Jiangsu 226000; ${ }^{3}$ Department of Radiotherapy Oncology, Chang An Hospital, Xi'an, \\ Shaanxi 710010; ${ }^{4}$ Cancer Centre, Institute of Translational Medicine, Faculty of Health Sciences, \\ University of Macau, Taipa, Macao SAR 999078, P.R. China
}

Received June 9, 2020; Accepted August 6, 2020

DOI: $10.3892 /$ wasj.2020.63

\begin{abstract}
Anti-PD-1 and PD-L1 inhibitors are associated with several adverse events, including a spectrum of immune-related adverse effects (irAEs). Neurologic irAEs are uncommon occurrences with varied presentations. The present study presents a case of facial nerve injury irAEs associated with adjuvant pembrolizumab therapy $\left(\right.$ Keytruda $\left.{ }^{\circledR}\right)$, which exhibited unusual presentations. A 44-year-old male patient with extensive-stage small-cell lung cancer (ES-SCLC) was treated with a 6-cycle etoposide and cisplatin (EP) regimen followed by $200 \mathrm{mg}$ pembrolizumab $\left(\right.$ Keytruda $^{\circledR}$ ) intravenously once every 3 weeks. Magnetic resonance imaging (MRI) imaging of the brain revealed that there were abnormal enhancement areas in the left pontine crus, with clear boundaries. The patient terminated the use of pembrolizumab; however, EP chemotherapy continued and he was treated with high-dose hormone therapy with the resolution of neurological symptoms. A total of $40 \mathrm{mg}$ methylprednisolone was administered for 5 days and the patient
\end{abstract}

Correspondence to: Dr Shigao Huang, Cancer Centre, Institute of Translational Medicine, Faculty of Health Sciences, University of Macau, University Avenue, Taipa, Macao SAR 999078, P.R. China E-mail: huangshigao2010@aliyun.com

Dr Tao Qi, Department of Radiation Oncology, 986 Hospital Affiliated to The Fourth Military Medical University, 6 Jianshe West Road, Xi'an, Shaanxi 710054, P.R. China

E-mail: 395253175@qq.com

*Contributed equally

Abbreviations: ES-SCLC, extensive-stage small-cell lung cancer; $\mathrm{EP}$, etoposide and cisplatin; ICIs, immunity checkpoints inhibitors; irAEs, immune-related adverse effects; MRI, magnetic resonance imaging; PD-1, programmed cell death protein 1

Key words: cancer, immunotherapy, pembrolizumab, immunerelated adverse events, neurotoxicity, facial nerve injury was then administered oral prednisone tablets $(25 \mathrm{mg})$. After 1 week, the facial symptoms were significantly alleviated. Following hormone therapy, the re-examination of head MRI revealed that the lesion had shrunk and the symptoms had improved. The patient remains disease-free. Facial nerve injury of neurological irAEs is uncommon adverse events in the context of PD-1 inhibitor therapy. The neurotoxicity caused by immune checkpoint inhibitors (ICIs) should be a cause of concern. The early recognition of neurological irAEs is critical for the initiation of specific anti-inflammatory agents for the prevention of such events. These events are usually alleviated with hormone therapy, which can distinguish metastatic tumors from neuropathy.

\section{Introduction}

PD-1 is expressed on the surface of a variety of immune cells, such as T cells, B cells and natural killer (NK) cells, in which its ligand, programmed death-ligand (PD-L)1, is widely expressed on the surface of various tissue cells, including tumor cells, while the ligand PD-L2 is mainly expressed in hematopoietic cells. PD-L1 is one of the numerous immune checkpoints of T cells, and the PD-L1 signaling pathway inhibits the activity of $\mathrm{T}$ cells at a later stage of the immune response $(1,2)$. PD1 monoclonal antibodies, including pembrolizumab and nivolumab, block PD-1/PD-L1 association and enhance the anti-tumor immune function of $\mathrm{T}$ cells.

Pembrolizumab is one of the immune checkpoint inhibitors (ICIs), which is a humanized monoclonal $\mathrm{IgG}$, applied in the immune therapy of lung cancer (3), melanoma (4), renal cancer (5) and other malignant tumors (6). The survival benefit is evident (7). Immune inhibition release leads to immune-related side-effects, such as pembrolizumab, a PD-1 inhibitor with median toxicity of $77.1 \%$. The incidence of toxicity for above grade 3 is $20.8 \%$. The overall immunotherapy is safe; however, some of the fatal immunotoxicities, such as neurological adverse events (nAEs) associated with ICIs should attract sufficient attention, particularly when neurotoxicity is involved. According to a previous study, the incidence of neurological toxicity associated with the administration 
of PD-1 inhibitors is $6.1 \%$ (8), and neurotoxicity accounts for approximately $15 \%$ of the deaths caused by PD-1/PD-L1 inhibitors (9).

\section{Case report}

A 44-year-old male was diagnosed with extensive-stage small-cell lung cancer (ES-SCLC) accompanied by bone, brain and mediastinal lymph node metastasis. Between March, 2019 to August, 2019, he received systemic treatment with 6-cycle etoposide and cisplatin (EP) chemotherapy (etoposide $165 \mathrm{mg} / \mathrm{m}^{2}$ on days $1-3$, and cisplatin $50 \mathrm{mg} / \mathrm{m}^{2}$ on days $1-2$ ) followed by $200 \mathrm{mg}$ pembrolizumab (Keytruda ${ }^{\circledR}$, Merck Sharp \& Dohme Corp.) intravenously once every 3 weeks, and the curative efficacy was evaluated as progressive disease (PD). He arose with facial paralysis, facial numbness, pain, tinnitus and limb joint pain on September 23, 2019 (Fig. 1A), although he had tendon reflexes. An enhanced cranial MRI examination revealed that there were abnormal enhancement areas in the left pontine crus, with clear boundaries (Fig. 2A). Facial nerve thickening and swelling caused facial paralysis. According to the toxicity grade, it was classified as grade 2 . Thus, the use of pembrolizumab was terminated, although EP chemotherapy continued and he was treated with high-dose hormone therapy with the resolution of neurological symptoms. Methylprednisolone at $40 \mathrm{mg}$ was administered for 5 days and he was then treated with oral prednisone tablets (25 mg). After 1 week, his facial symptoms were significantly alleviated (Fig. 1B). Following hormone therapy, the re-examination of the head MRI revealed that the lesion had shrunk and symptoms had improved (Fig. 2B). Subsequently, he was treated with etoposide/tenoposide alone, and he did not exhibit any signs of neurotoxicity. After completing the whole chemotherapeutic regimen, the primary tumor had markedly shrunk when comparing pre-treatment (Fig. 3A) and post-treatment (Fig. 3B).

\section{Discussion}

The common pembrolizumab-associated neurological immune-related adverse effects (irAEs) span diverse entities, including Guillain barre syndrome, transverse myelitis, myasthenia gravis, peripheral neuropathy and aseptic meningitis (3). The presents study reports one case of pembrolizumab-associated neurological irAEs manifesting as pain, numbness and corner nakedness in the area of left facial nerve innervation, no tinnitus and hearing loss. When enquiring about the detailed history of the patient, a comprehensive detection of the nervous system, brain MRI, a cerebrospinal fluid examination, neurological analysis and consultation with a neurologist, tumor progression, transfer, central nervous system infection, diabetic neuropathy, or vitamin B12 deficiency-associated disease were excluded. Given the specification of glucocorticoid treatment underlying neurotoxicity, the symptoms and related image data markedly improved, and the patient was considered to have suffered from facial nerve toxicity caused by pembrolizumab. It has been reported that pembrolizumab-associated neurological irAEs occur both in peripheral neuropathy and
Guillain barre syndrome (5), although facial nerve injury is rare. The present case report demonstrated that there were marked individual differences in immune-related neurotoxic manifestations, which increases the difficulty in the early identification of toxic symptoms. The facial nerve is responsible for muscle movements in the face, and pembrolizumab-associated neurological irAEs cause facial deformation.

Although the compliance of ICI therapy with irAEs is affected, it does not seem to affect the efficacy of immunotherapy (6), and immune-related toxicity may be an index for the reactivity of ICI treatment and survival benefits $(7,8)$. A previous study (9) demonstrated that ICI therapy for patients with rheumatoid arthritis (RA) had a better response rate (85.7 vs. $35.3 \%$ ) than those who were negative for RA. Similarly, a preliminary study revealed that ICI treatment with neurotoxicity may have a better response rate (10). This may be one reason for the better treatment response in the present case.

irAEs require early identification and timely and effective drug intervention through NCCN guidelines (11). Glucocorticoids are the major drug choices; however, it has been shown that patients with immune-related neurotoxicity have different individual responses to glucocorticoids (12), and patients with resistance to glucocorticoids often need to be replaced with (TNF-a) infliximab or (IVIG) immunoglobulin. The patient, in this case (12), had a good response to glucocorticoids, and the symptoms of facial paralysis and pain in 4 joints were significantly improved following $72 \mathrm{~h}$ of administration, without hormone resistance.

The mechanism of toxicity associated with ICIs has not yet been fully clarified. It has been demonstrated that the following main categories exist: First, T cells directly attack normal tissues, and certain antigens can be expressed in both tumor cells and normal cells; thus, the enhanced function of T cells can cause damage to normal tissues (13). Another type of injury involves inflammatory factors, such as pro-inflammatory factor IL-17, which can be released with the recovery of T cell function $(14,15)$. Another type is that following treatment with ICI, the body's pre-existing autoreactive antibodies can be activated, leading to the occurrence of irAEs (16). In addition, the mechanisms of irAEs occurring in different phases may also differ. For example, the early side-effects are mainly manifested by extensive epithelial cell damage caused by the involvement of inflammatory cells, such as neutrophils, such as skin rash, colitis, pneumonia and other symptoms. The late side-effects are more likely to occur in the specific local organs, such as nervous system toxicity, hypophysitis. In the present case report, the patient facial nerve toxicity occurred following the end of ICI therapy, which is an advanced toxic reaction, and the specific mechanisms remain to be further elucidated.

In the present study, a case of a rare patient with ES-SCLC treated with anti-PD-1 inhibitors (pembrolizumab) with induced facial toxicity is reported, in order to provide some references for the prediction and management of the side-effects caused by ICIs.

In conclusion, the facial nerve injury caused by ICIs is rare; thus, the neurotoxicity caused by ICIs should be a matter of concern, and the related mechanisms underlying the injury 

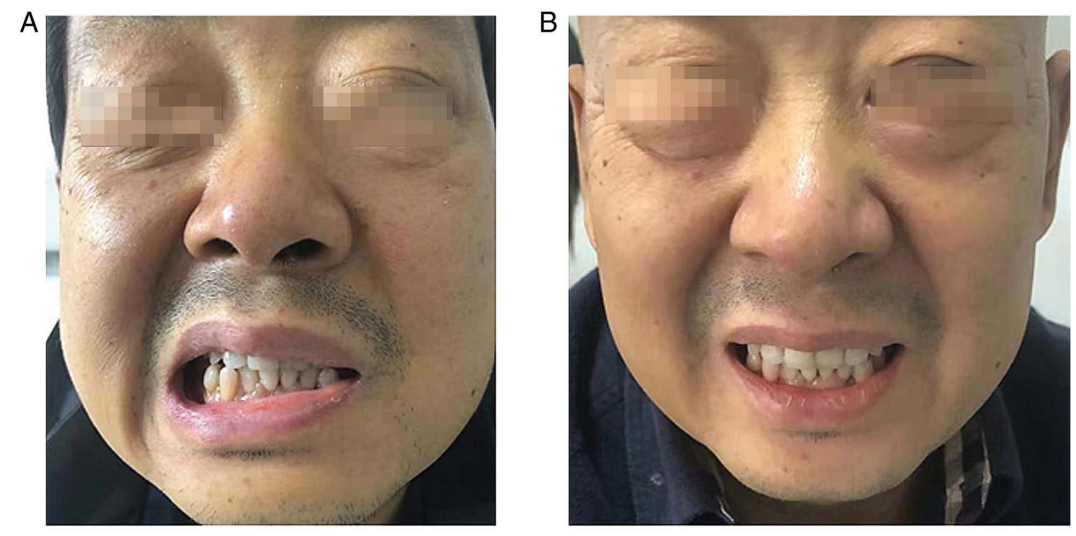

Figure 1. The patient arose with (A) facial paralysis, facial numbness, pain, tinnitus, and limb joint pain. (B) After 1 week his facial symptoms were significantly alleviated.
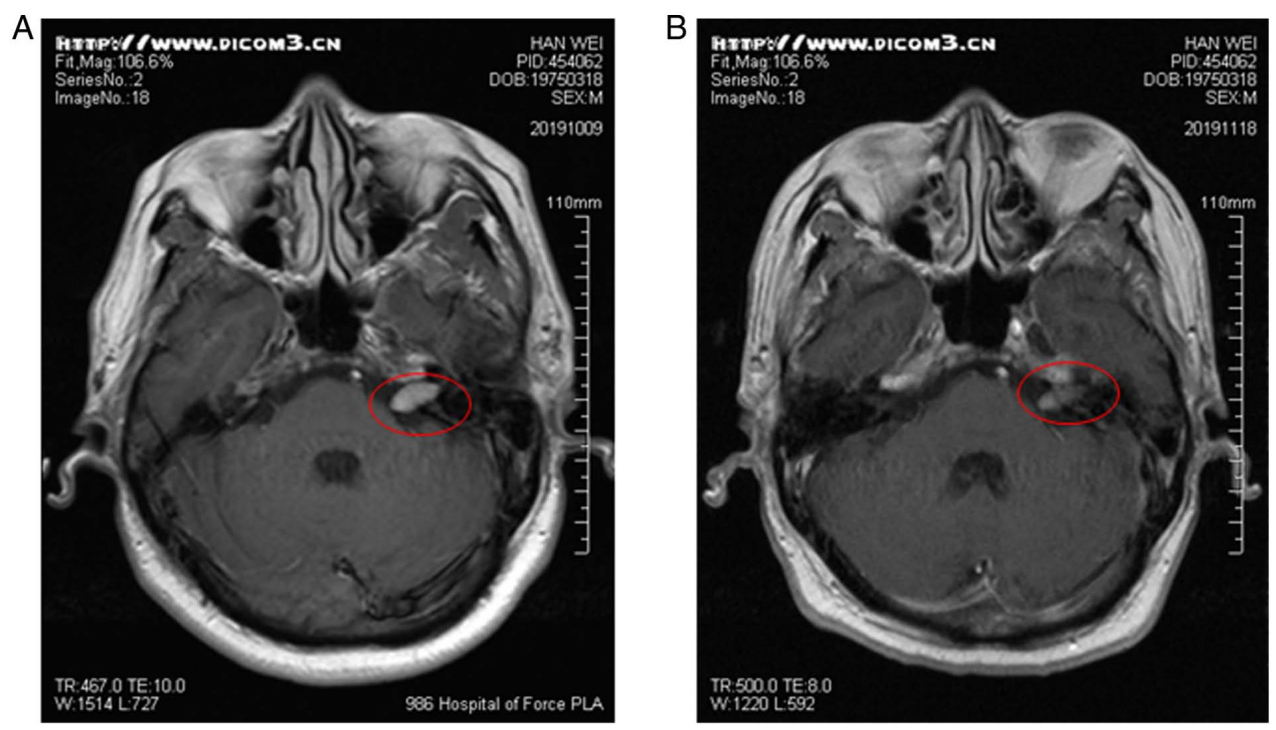

Figure 2. Re-examination of the head MRI revealed (A) facial nerve thickening and swelling caused facial paralysis. (B) Following hormone therapy, the lesion had shrunk and the symptoms had improved.

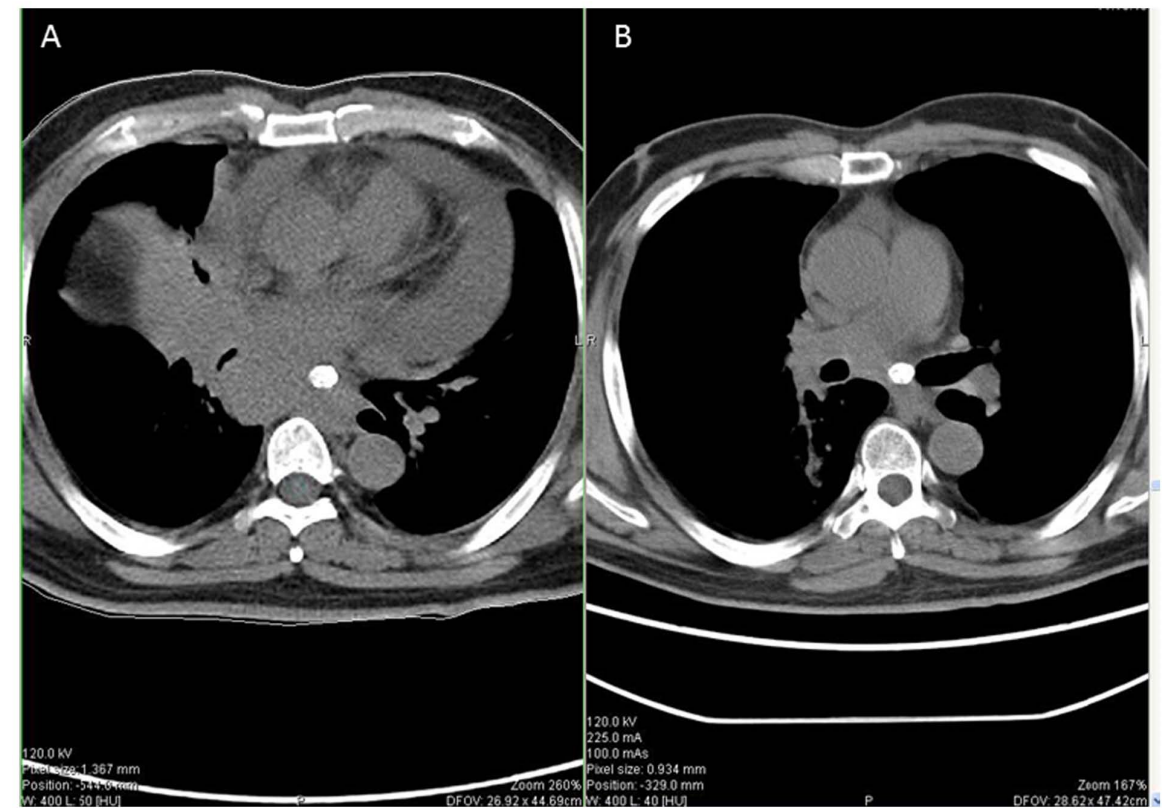

Figure 3. The lung tumor markedly shrank sharply when comparing (A) before immunotherapy with (B) after immunotherapy. 
warrant further investigation. While chemotherapy does not cause such nerve damage, immune-induced nerve damage is usually alleviated with hormone therapy, which can distinguish metastatic tumors from neuropathy.

\section{Acknowledgements}

Not applicable.

\section{Funding}

No funding was received.

\section{Availability of data and materials}

All data generated or analyzed during this study are included in this published article or are available from the corresponding author on reasonable request.

\section{Authors' contributions}

YD and SH conceived the case report, wrote the initial manuscript and reviewed the final manuscript. MW, WL, SH, HG and TQ interpreted and created the radiographic images, and reviewed the final manuscript. All authors read and approved the final manuscript.

\section{Ethics approval and consent to participate}

The present study was approved by the 986 Hospital Affiliated to the Fourth Military Medical University Ethics Board (approval no. KJ4-004-03; Xi'an, China). Consent to participate was obtained from the patient.

\section{Patient consent for publication}

Informed written consent was obtained from the patient for publication of this case.

\section{Competing interests}

The authors declare that they have no competing interests.

\section{References}

1. Boussiotis VA: Molecular and biochemical aspects of the PD-1 checkpoint pathway. N Engl J Med 375: 1767-1778, 2016.

2. Coles SJ, Gilmour MN, Reid R, Knapper S, Burnett AK, Man S, Tonks A and Darley RL: The immunosuppressive ligands PD-L1 and CD200 are linked in AML T-cell immunosuppression: Identification of a new immunotherapeutic synapse. Leukemia 29: 1952-1954, 2015.

3. Lim SH, Sun JM, Lee SH, Ahn JS, Park K and Ahn MJ: Pembrolizumab for the treatment of non-small cell lung cancer. Expert Opin Biol Ther 16: 397-406, 2016.
4. Robert C, Ribas A, Schachter J, Arance A, Grob JJ, Mortier L, Daud A, Carlino MS, McNeil CM, Lotem M, et al: Pembrolizumab versus ipilimumab in advanced melanoma (KEYNOTE-006): Post-hoc 5-year results from an open-label, multicentre, randomised, controlled, phase 3 study. Lancet Oncol 20: 1239-1251, 2019.

5. Rini BI, Plimack ER, Stus V, Gafanov R, Hawkins R, Nosov D, Pouliot F, Alekseev B, Soulières D, Melichar B, et al: Pembrolizumab plus axitinib versus sunitinib for advanced renal-cell carcinoma. N Engl J Med 380: 1116-1127, 2019.

6. Fradet Y, Bellmunt J, Vaughn DJ, Lee JL, Fong L, Vogelzang NJ, Climent MA, Petrylak DP, Choueiri TK, Necchi A, et al: Randomized phase III KEYNOTE-045 trial of pembrolizumab versus paclitaxel, docetaxel, or vinflunine in recurrent advanced urothelial cancer: Results of $>2$ years of follow-up. Ann Oncol 30: 970-976, 2019.

7. Gao HX, Huang SG, Du JF, Zhang XC, Jiang N, Kang WX, Mao $\mathrm{J}$ and Zhao Q: Comparison of prognostic indices in NSCLC patients with brain metastases after radiosurgery. Int $\mathrm{J}$ Biol Sci 14: 2065-2072, 2018.

8. Cuzzubbo S, Javeri F, Tissier M, Roumi A, Barlog C, Doridam J, Lebbe C, Belin C, Ursu R and Carpentier AF: Neurological adverse events associated with immune checkpoint inhibitors: Review of the literature. Eur J Cancer 73: 1-8, 2017.

9. Wang DY, Salem JE, Cohen JV, Chandra S, Menzer C, Ye F, Zhao S, Das S, Beckermann KE, Ha L, et al: Fatal toxic effects associated with immune checkpoint inhibitors: A systematic review and meta-analysis. JAMA Oncol 4: 1721-1728, 2018.

10. Feng S, Coward J, McCaffrey E, Coucher J, Kalokerinos P and O'Byrne K: Pembrolizumab-induced encephalopathy: A review of neurological toxicities with immune checkpoint inhibitors. J Thorac Oncol 12: 1626-1635, 2017.

11. Thompson JA, Schneider BJ, Brahmer J, Andrews S, Armand P, Bhatia S, Budde LE, Costa L, Davies M, Dunnington D, et al: Management of immunotherapy-related toxicities, version 1.2019. J Natl Compr Canc Netw 17: 255-289, 2019.

12. Garcia CA, El-Ali A, Rath TJ, Contis LC, Gorantla V, Drappatz J and Davar D: Neurologic immune-related adverse events associated with adjuvant ipilimumab: Report of two cases. J Immunother Cancer 6: 83, 2018.

13. Johnson DB, Balko JM, Compton ML, Chalkias S, Gorham J Xu Y, Hicks M, Puzanov I, Alexander MR, Bloomer TL, et al: Fulminant myocarditis with combination immune checkpoint blockade. N Engl J Med 375: 1749-1755, 2016.

14. Tarhini AA, Zahoor H, Lin Y, Malhotra U, Sander C, Butterfield LH and Kirkwood JM: Baseline circulating IL-17 predicts toxicity while TGF- $\beta 1$ and IL-10 are prognostic of relapse in ipilimumab neoadjuvant therapy of melanoma. J Immunother Cancer 3: 39, 2015.

15. Feng T, Qin H, Wang L, Benveniste EN, Elson CO and Cong Y: Th17 cells induce colitis and promote Th1 cell responses through IL-17 induction of innate IL-12 and IL-23 production. J Immunol 186: 6313-6318, 2011.

16. Osorio JC, Ni A, Chaft JE, Pollina R, Kasler MK, Stephens D, Rodriguez C, Cambridge L, Rizvi H, Wolchok JD, et al: Antibody-mediated thyroid dysfunction during T-cell checkpoint blockade in patients with non-small-cell lung cancer. Ann Oncol 28: 583-589, 2017. 